\title{
Janet Hyde (1986)
}

\author{
Encyclopedia Entry by Katherine S. Corker
}

Version as of March 2, 2016

For T.K. Shackelford \& V.A. Weekes-Shackelford (Eds.) (2019). Encyclopedia of evolutionary psychological science. Switzerland: Springer International Publishing.

DOI of published version: 10.1007/978-3-319-16999-6_847-1

\section{Synonyms}

Janet Hyde (2005); Janet Hyde (2014); Janet Hyde (1984)

\section{Definition}

An early meta-analysis showing that gender differences in aggressive behavior are moderate in size but persistent, yet decreasing in magnitude, over the lifespan.

\section{Introduction}

Are men and boys more aggressive than women and girls? Janet Hyde (1986) conducted the first modern meta-analysis concerning this question. Meta-analysis is a quantitative statistical technique in which a researcher seeks to compute an estimate of an effect size. The effect size that Hyde (1986) sought to quantify was the magnitude of the gender difference in aggression, including physical aggression and verbal aggression. The effect size that Hyde used was Cohen's $d$, a standardized effect size. A standardized effect size is a ratio of the magnitude of the difference between groups, relative to how much individuals within a group vary from one another. Positive effect size estimates indicate that men and boys have higher levels of aggression than women and girls, and effect sizes of $0.20,0.50$, and 0.80 are considered small, medium, and large differences, respectively (Cohen, 1988). 
Building on her previous work (Hyde, 1984) stemming from Maccoby and Jacklin's (1974) review, Hyde (1986) sought to apply newer meta-analytic techniques to the question. In particular, Hyde was concerned with testing and accounting for heterogeneity: the notion that characteristics of studies might be associated with differences in effect size estimates. The characteristics she examined were type of aggression, method of measurement, study design, and age of participants.

\section{Type of Aggression}

An important question in the literature historically has been whether there is a gender difference in aggression, depending on the type of aggression investigated (physical vs. verbal). Hyde reported that although the effect size for physical aggression appeared larger $(d=0.60$, based on 26 studies) than the effect size for verbal aggression ( $d=0.43$, based on 6 studies), this difference was not statistically significant. Both effect size estimates were positive, indicating that men and boys showed higher levels of both physical and verbal aggression than women and girls.

More recently, researchers have asked whether women and girls show more indirect or relational aggression than men and boys. Indirect aggression differs from direct (verbal and physical) aggression in that when directly aggressing, the perpetrator of aggression directly engages a victim, whereas with indirect aggression, the perpetrator seeks to harm the victim by damaging a victim's reputation. A more recent meta-analysis (Archer, 2004) found a small negative effect size such that women and girls were more indirectly aggressive than men and boys, but this newer meta-analysis also replicated Hyde (1986), finding a positive effect size of moderate size for both physical and verbal direct aggression.

\section{Method of Measurement}


The method of assessment of aggression varied across studies in Hyde's (1986) metaanalysis. Most studies used direct observation (42 studies), whereas other studies used self-report (14 studies), parent or teacher report (8 studies), projective assessment (1 study), or peer report (4 studies). Hyde (1986) reported significant heterogeneity based on method of assessment, implying that there were differences in the effect sizes observed depending on measurement method. However, the effect size for self-report $(d=0.40)$ was not statistically smaller than the effect size for direct observation $(d=0.51)$.

\section{Study Design}

Hyde (1986) contrasted observational and correlational studies with laboratory experiments. In a true experiment, the researcher manipulates and controls an independent variable (in this case, usually a provocation to aggress) and observes its effects on a dependent variable (here, an aggressive behavior or cognition). In observational/correlational studies, a researcher tests for an association between measured variables - in this case, typically gender and a self-report of unprompted aggressive thoughts or behavior.

In her meta-analysis, Hyde (1986) reported that effect sizes were larger in observational/correlational studies than experimental studies, with effect sizes of $d=0.56$ (a moderate effect size) in 42 studies vs. $d=0.29$ (a small to moderate effect size) in 27 studies.

\section{Developmental Effects}

Hyde (1986) reported that gender differences decreased in magnitude over the course of development. That is, gender differences were larger among pre-school aged children $(d=0.58)$ than they were among college students $(d=0.27)$.

It is noteworthy that in a more recent review of over 350 studies, Archer (2004) reported effect sizes of 0.56 for elementary school aged children (ages 6-11), compared to 0.29 for 
emerging adults (aged 22-30 years), and -0.01 for adults over age 31. Importantly, Hyde (1986) explained that methodological factors in different studies (e.g., method of measurement, type of aggression studied) are confounded with age of sample, making firm developmental conclusions difficult.

\section{Conclusion}

Janet Hyde's (1986) meta-analysis was a groundbreaking work in early investigations of gender differences in aggression. The paper is important because it was one of the first to use newly developed tests of heterogeneity to determine whether effect size estimates for gender differences varied based on type of aggression, method of measurement, study design, and developmental period. Hyde reported that effect size estimates did vary based on study design and developmental period, but there were no significant differences between physical and verbal aggression, nor between studies assessing aggression using self-report vs. direct observation. Overall, estimates were moderate in size and positive in magnitude, indicating support for the notion that men and boys are generally more aggressive than women and girls.

From a modern vantage point, Hyde (1986) leaves some important questions unanswered. In particular, it fails to correct effect size estimates for publication bias, a well-known cause of overestimation in effect size. Archer (2004) reported some attempts to assess publication bias in this literature, but an update of this meta-analysis with more modern bias correction techniques would be warranted at this point.

\section{Cross-References}

Female-Perpetrated Violence; Benefits of Aggression; Context-Specificity of Aggression; Costs of Aggression; Men Riskier, More Aggressive; Sex Differences in Aggression; Aggression; Aggressive Fantasies; Imitative Aggression; Meta-Analysis of Sex Differences in Aggression; 
Physical Aggression; Social vs. Physical Aggression; Young Men Most Risky, Aggressive;

Development of Aggression; Indirect Aggression; Relational Aggression; Gender Symmetry and Sociocultural Theories of Violence

\section{References}

Archer, J. (2004). Sex differences in aggression in real-word settings: A meta-analytic review. Review of General Psychology, 8, 291-322.

Cohen, J. (1988). Statistical power analysis for the behavioral sciences ( $2^{\text {nd }}$ ed.). Hillsdale, NJ: Erlbaum.

Hyde, J. S. (1984). How large are gender differences in aggression? A developmental metaanalysis. Developmental Psychology, 20, 722-736.

Hyde, J. S. (1986). Gender differences in aggression. In J. S. Hyde \& M. C. Linn (Eds.) The psychology of gender: Advances through meta-analysis (pp. 51-66). Baltimore, MD: The Johns Hopkins University Press.

Hyde, J. S. (2005). The gender similarities hypothesis. American Psychologist, 60, 581-592.

Hyde, J. S. (2014). Gender similarities and differences. Annual Review of Psychology, 65, 373398.

Maccoby, E. E., \& Jacklin, C. N. (1974). The psychology of sex differences. Stanford, CA: Stanford University Press. 